\title{
A New Serum Antibody Test Kit (E Plate) for Evaluation of Helicobacter Pylori Eradication
}

\author{
Takashi Kawai, Kohei Kawakami, Taku Kudo, Shoji Ogiahara, Yutaka Handa and Fuminori Moriyasu
}

\begin{abstract}
Objective Serological antibody test have been widely performed to detect the presence of $\mathrm{H}$. pylori, but they have not been used to evaluate the status of $\mathrm{H}$. pylori after eradication. In this study we evaluated the diagnostic accuracy of a new serological test kit (E-plate) after eradication.

Method Eradication of $\boldsymbol{H}$. pylori was performed in $\mathbf{1 0 0}$ patients by proton pump inhibitor (PPI)+amoxicillin (AMPC)+clarithromycin (CAM) or PPI+AMPC therapy. Evaluation of $\boldsymbol{H}$. pylori was done by culture, histology and rapid urease test before, and 8 weeks after, the treatment. Serological tests were also performed before and after treatment using the $E$ plate. Cure was defined as no evidence of $H$. pylori at 8 weeks after the treatment. Receiver operating characteristic (ROC) analysis was performed to determine the ideal cut-off value for percentage change in the serological test.

Result Success was obtained in $\mathbf{7 3}$ patients, failure in $\mathbf{2 0}$ patients and there were 7 dropouts. Serological test value was significantly decreased after treatment $(44.3 \pm 29.6 \mathrm{U} /$ $\mathrm{ml})$ compared to before treatment $(94.8 \pm 73.2 \mathrm{U} / \mathrm{ml})$ in the successful cases. In contrast, those with no significant change after treatment $(62.7 \pm 31.3 \mathrm{U} / \mathrm{ml})$ compared to before treatment $(72.9 \pm 47.7 \mathrm{U} / \mathrm{ml})$ were considered as failure cases. ROC analysis revealed that cut-off values of a $20 \%$, $30 \%$, and $40 \%$ decrease on $E$ plate result yielded a sensitivity of $95.5 \%, 92.4 \%, 71.2 \%$ and a specificity of $73.3 \%$, $84.2 \%, 94.7 \%$, respectively.

Conclusion The new $\mathbf{E}$ plate serological test kit for $\boldsymbol{H}$. pylori was useful for distinguishing success from failure 8 weeks after completion of eradication therapy for $H$. pylori.
\end{abstract}

(Internal Medicine 41: 780-783, 2002)

Key words: Helicobacter pylori, serological test, pepsinogen

\section{Introduction}

Helicobacter pylori ( $H$. pylori) has been shown to be related to peptic ulcers, chronic gastritis, MALT lymphoma and gastric cancer (1-4). Recurrence of gastric and duodenal ulcers can be significantly prevented by its eradication (2). In Japan, H. pylori eradication therapy in patients with gastric and duodenal ulcers has been included in health insurance coverage since November 2000. Diagnostic methods for detecting H. pylori infection can be divided into invasive and non-invasive methods. The former methods can be classified into the culture, rapid urease test, and histological examination using upper gastrointestinal endoscope, while the latter methods include the urea breath test and antibody determination method (using serum or urine samples). Although the non-invasive serum antibody determination method is the most common at present, it has not been used in the evaluation of eradication of H. pylori infection. In the present study we evaluated the usefulness of the E Plate, a new serum antibody kit developed in Japan, in the assessment of $H$. pylori eradication therapy.

\section{Subjects and Methods}

The subjects consisted of 100 consecutive patients who were found to be $H$. pylori-positive by upper GI endoscope between October 1999 and June 2000. There were 65 men and 35 women aged $53.2 \pm 11.6$ years on the average. The 100 subjects included 27 gastric ulcer cases (or ulcer scar), 20 duodenal ulcer cases (or ulcer scar), 9 gastro-duodenal ulcer cases (or ulcer scar), 40 chronic gastritis cases and 4 gastric polyp cases.

The study protocol was approved by the hospital clinical ethics committee on human experiments and with the Helsinki Declaration of 1975, as revised in 1983. After obtaining written informed consent, the patients were randomly assigned to one of the following 4 groups: 1) the RA Group, receiving a 2week course of $20 \mathrm{mg} /$ day rabeprazole (RPZ) once a day and $500 \mathrm{mg}$ of amoxicillin (AMPC) 4 times a day, 2) the OAC Group, receiving a 1-week course of $20 \mathrm{mg} /$ day omeprazole (OPZ) once a day, $500 \mathrm{mg}$ AMPC 3 times a day, and $200 \mathrm{mg}$

From the Fourth Department of Internal Medicine, Tokyo Medical University, Tokyo

Received for publication September 5, 2001; Accepted for publication June 4, 2001

Reprint requests should be addressed to Dr. Takashi Kawai, the fourth Department of Internal Medicine, Tokyo Medical University, 6-7-1 Nishishinjuku, Shinjuku-ku, Tokyo 160-0023 
clarithromycin (CAM) 3 times day, 3) the LAC Group, receiving a 1-week course of $30 \mathrm{mg}$ lansoprazole (LPZ) once a day together with AMPC and CAM in the same way as in the OAC Group, and 4) the RAC Group, receiving a 1-week course of $10 \mathrm{mg}$ of RPZ once a day together with AMPC and CAM in the same way as in the OAC Group. A third party-controlled central management system was employed for medication regardless of the diagnosis or severity of the disease.

\section{Evaluation of $\boldsymbol{H}$. pylori infection}

Three biopsy specimens each were obtained endscopically from the greater curvature at the gastric antrum and body (total 6 specimens) in order to determine the presence or absence of $H$. pylori infection before starting eradication therapy and 8 weeks after its completion. Each specimen was examined by the rapid urease test (CLO test; Delta West Pty Ltd., Bentley, WA, Australia), culture and histological examination. For histological examinations, specimens were fixed in formalin and stained with hematoxylin and eosin and also Giemsa in order to determine the existence of $H$. pylori under an optical microscope. For culture studies, biopsy specimens of the gastric mucosa was homogenized, and the homogenate was inoculated to Skirrow's medium (Nissui Seiyaku Company Ltd., Tokyo) for culture for 5 to 7 days under microaerophilic conditions at $37^{\circ} \mathrm{C}\left(\mathrm{O}_{2} 5 \%, \mathrm{CO}_{2} 10 \%, \mathrm{~N}_{2} 85 \%\right)$. Eradication therapy was regarded as successful if the results of culture, histological examination and RUT were all negative.

\section{Evaluation of serum $\mathrm{H}$. pylori antibody titer and serum pepsi- nogen levels}

Blood samples were taken from patients at the time of endoscopy before eradication therapy and 8 weeks after its completion, and serum was separated to determine $H$. pylori antibody and pepsinogen I and II levels (PGI and PGII) by microplate EIA using an antibody determination kit (E Plate Eiken $H$. pylori Antibody, Eiken Chemical Co., Ltd., Tokyo, hereafter referred to as E Plate) and a microplate EIA pepsinogen kit (E Plate Eiken Pepsinogen I and II, Eiken Chemical Co., Ltd., Tokyo), respectively. Strains isolated from Japanese patients with gastric ulcers were used as antigens to prepare $\mathrm{E}$ plate. Furthermore, the total protein fraction, including highand low-molecular weight (10,000-500,000 Da) fractions, obtained from the supernatant obtained by ultrasonic ultracentrifugation of bacteria was used as the antigen.

The plate solid phase 2-step sandwich EIA method was employed. $H$. pylori-antigen solid phase plates were incubated after adding buffer solution, followed by the standard $H$. $p y$ lori antibody or specimen. The reaction fluid was removed by washing and enzyme-labeled anti-human IgG antibody was introduced for further incubation. After reaction, non-reactive enzyme-labeled anti-human IgG antibody was removed by washing, and the enzymatic reaction was conducted after adding a substrate fluid. Coloration was determined using a spectrophotometer for microplates, and a standard curve was drawn based on the absorbance values obtained to determine $H$. $p y$ lori antibody concentrations. Pepsinogen was determined in the same way, and the pepsinogen I/II ratio (PEG I/II) was calculated.

Percent changes before and after eradication were calculated using the following equation: (value obtained 8 weeks after eradication-pre-eradication baseline)/pre-eradication baseline $\times 100$. In the $E$ plate method, the cut-off value was set based on the receiver operating characteristic (ROC) curve to determine whether or not eradication was achieved. Percent changes in pepsinogen were used to determine whether or not eradication was achieved according to the method of Furuta et al (5). The results obtained by E plate and PEG I/II were compared using specimens obtained endoscopically.

\section{Statistical analysis}

Statistical analysis was conducted by student's t-test, and a P-value of less than 0.05 was regarded as indicating a statistically significant difference.

\section{Results}

Eradication therapy was successful in 73 patients and failure in 20 , with 7 dropouts. The per-protocol eradication rate was $78.5 \%$ (73/93), while the eradication rate determined by intention-to-treat (ITT) analysis was $73 \%$ (73/100). The perprotocol and ITT eradication rates in each group were as follows: $79.2 \%$ and $76.0 \%$, with success in 19 patients, failure in 5 and dropout in 1 in the RA Group; $82.6 \%$ and $76.0 \%$, with success in 19 patients, failure in 4 and dropout in 2 in the OAC Group; $74.0 \%$ and $68.0 \%$, with success in 17 patients, failure in 6 and dropout in 2 in the LAC Group; and $78.3 \%$ and $72.0 \%$, with success in 18 patients, failure in 5 and dropout in 2 in the RAC Group (Table 1). No significant differences were observed among the four regimens.

$H$. pylori antibody titer was determined before and after eradication therapy in 66 and 19 patients in whom eradication was successful and failure, respectively. The reason for nondetermination was failure to collect of serum samples before eradication therapy for patients' personal reasons. The $H$. $p y$ lori antibody titer decreased significantly from 94.8 $\pm 73.2 \mathrm{U} /$

Table 1. Cure Rate of Each Regimen

\begin{tabular}{lcc}
\hline & ITT & PP \\
\hline RA & $19 / 25(76 \%)$ & $19 / 24(79.2 \%)$ \\
OAC & $19 / 25(76 \%)$ & $19 / 23(82.6 \%)$ \\
LAC & $17 / 25(68 \%)$ & $17 / 23(74.0 \%)$ \\
RAC & $18 / 25(72 \%)$ & $18 / 23(78.3 \%)$ \\
& & \\
total & $73 / 100(73 \%)$ & $73 / 93(78.5 \%)$ \\
\hline
\end{tabular}

ITT: intention to treat analysis, PP: per protocol analysis, RA: rabeprazole (RPZ)+amoxicillin (AMPC), RAC: RPZ+AMPC+clarithomycin (CAM), LAC: lansoprazole+AMPC+CAM, OAC: omeprazole+AMPC+CAM. 
Table 2. Change of Value of IgG $\boldsymbol{H}$. pylori Antibody (E plate) before and 8 weeks after Completion of Eradication in Success and Failure

\begin{tabular}{lcc}
\hline & Success $(\mathrm{U} / \mathrm{ml})$ & Failure $(\mathrm{U} / \mathrm{ml})$ \\
\hline before & $94.8 \pm 73.2^{*}$ & $72.9 \pm 47.7$ \\
after & $44.3 \pm 29.6$ & $62.7 \pm 31.3$ \\
\hline
\end{tabular}

$* \mathrm{p}<0.05$

Table 3. Change of Value Pepsinogen I/II Ratio before and 8 wks after Completion of Eradication in Success and Failure

\begin{tabular}{lll}
\hline & Success & Failure \\
\hline before & $2.66 \pm 1.10^{*}$ & $3.01 \pm 1.16$ \\
after & $5.19 \pm 1.86$ & $3.35 \pm 1.40$ \\
\hline
\end{tabular}

$* \mathrm{p}<0.01$.

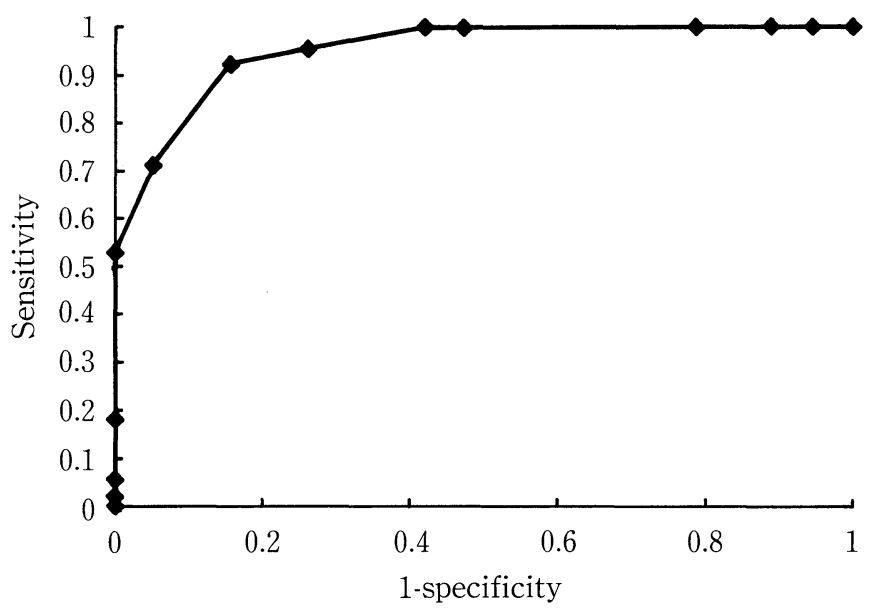

Figure 1. Receiver operating characteristic (ROC) curve for evaluation of eradication using IgG $\mathrm{H}$. pylori antibody (E plate).

$\mathrm{ml}$ before therapy to $44.3 \pm 29.6 \mathrm{U} / \mathrm{ml}$ after therapy in patients in whom the therapy was successful, while no significant differences were seen before $(72.9 \pm 47.7 \mathrm{U} / \mathrm{ml})$ and after $(62.7 \pm 31.3 \mathrm{U} / \mathrm{ml})$ therapy in patients in whom it was failure (Table 2). The PEG I/II ratio increased significantly from $2.66 \pm 1.10$ before therapy to $5.19 \pm 1.86$ after therapy in the successful group. A slighter increase was observed in the failure group from $3.01 \pm 1.16$ to $3.35 \pm 1.40$ (Table 3 ).

The ROC curve for percent changes in $H$. pylori antibody titer are illustrated in Fig. 1. The cut-off value was set at $-20 \%$, $-30 \%$ and $-40 \%$ based on the ROC curve to determine whether or not eradication was achieved. High agreement rates were obtained between this evaluation and the evaluation using biopsy materials: $95.5 \% / 73.7 \%, 92.4 \% / 84.2 \%$ and $71.2 \% / 94.7 \%$ (sensitivity/specificity), respectively, at the cut-off values of $20 \%,-30 \%$ and $-40 \%$. On the other hand, the agreement rate between evaluation based on percent changes in the PEG I/II ratio and the evaluation using biopsy materials was $98.4 \% /$ $84.2 \%$ (sensitivity/specificity).

\section{Discussions}

Serological diagnostic methods such as the serum H. pylori antibody method and the serum pepsinogen method have a number of advantages over other methods, such as being less invasive than endoscopic methods (culture, histological examinations and RUT), cheaper than the urea breath test, not requiring dietary restrictions before tests, and the possibility of treatment of a large number of specimens at a time. At present, various serum antibody determination kits are commercially available, but test results using these kits do not necessarily agree because they use different antigens. E Plate uses an antigen extracted from $H$. pylori strains isolated in Japan and requires only simple procedures because it does not require dilution of specimens. According to Fujioka and Tokieda (6), the relative sensitivity, specificity and agreement rates between results obtained using this plate and those obtained by culture/ RUT were $100 \%, 80.0 \%$ and $97.1 \%$, respectively. However, the serum antibody titer is reported to decrease gradually as eradication therapy is conducted, and at least one year is needed until negative conversion is achieved after eradication. Cutler et al (7) who monitored changes in serum antibody titer after eradication, reported that antibody titer decreases by $26 \%, 43 \%$ and 55\%, respectively, 3, 6 and 12 months after eradication. The relative sensitivity and specificity were $86 \%$ and $88 \%$, respectively, in the evaluation conducted when serum antibody cutoff titer was taken as a $20 \%$ decrease at 6 months after eradication. In the present study, we investigated whether it is possible to determine the presence or absence of $H$. pylori at an earlier stage after eradication using the $\mathrm{E}$ plate.

In the present study, the $H$. pylori antibody titer decreased significantly 8 weeks after completion of eradication therapy, to about $50 \%$ of the pre-treatment baseline, in patients in whom H. pylori was eradicated. According to Kosunen et al (8), the antibody titer decreased by $50 \% 6$ months after eradication in $97 \%$ of patients in whom $H$. pylori was eradicated, while Wang et al (9) using a GAP-IgG kit, reported that the antibody titer decreased by at least one grade 6 months after eradication in 95\% of patients in whom $H$. pylori was eradicated. With many of the conventional antibody determination kits, decreases in antibody titer cannot be determined until a long time (6 months) after eradication of $H$. pylori. However, our findings showed that E Plate shows marked decreases in antibody titer even at 8 weeks after eradication is achieved.

According to the reports by Leung et al (10) and Miwa et al (11) the diagnostic accuracy of the serum antibody determination method developed in the West is not as good in Chinese and Japanese as it is in Caucasians. Decreases in the antibody 


\section{Helicobacter pylori and Serum Antibody}

titer observed at an early stage after bacterial eradication from E plates are thought to be related to the fact that strains isolated from Japanese were used as antigens to prepare the $\mathrm{E}$ plates.

Furuta et al (5), who compared the PEG I/II ratio determined by RIA one month after eradication with the pre-eradication baseline, reported that there were significant differences between patients in whom $H$. pylori was eradicated and those in whom it was not. Our findings obtained using the E Plate also shows similar changes in patients in whom $H$. pylori was eradicated. However, the ratio was slightly increased 8 weeks after eradication compared to the pre-eradication baseline in patients in whom $H$. pylori was not eradicated. Because the PEG I/II ratio reflects gastric mucosal inflammation, this increase may be attributable to the fact that eradication therapy transiently improves inflammation even if it does not completely eradicate $H$. pylori. In three patients in whom the diagnosis based on the PEG I/II ratio was false positive, histological findings showed a decrease of one grade or two grades in lymphocyte and neutrophil infiltration at the greater curvature in the gastric body compared to pre-eradication, indicating a slight improvement in inflammatory findings.

In the evaluation based on percent changes after eradication therapy compared to the pre-treatment baseline, the sensitivity and specificity of the E Plate method were $92.4 \%$ and $84.2 \%$, respectively, at a percent change of $-30 \%$. The sensitivity and specificity of the evaluation based on percent changes in the PEG I/II ratio were $98.4 \%$ and $84.2 \%$, respectively. Evaluation of eradication using the $E$ plate method is equivalent to that using the PEG I/II ratio, and the former is more accurate for detecting $H$. pylori infection.

Among various methods now available for the evaluation of eradication of $H$. pylori, histological examinations, culture, RUT, antibody determination and urea breath test (UBT) are covered by health insurance programs in Japan, but only UBT is effective for early determination (12). According to Nishikawa et al (13), the sensitivity of the RUT is very poor $(60.5 \%)$ when conducted 1 month after eradication although its specificity is high (99.2\%). Murata et al (14) also conducted a similar study by using by RUT and found that both the sensitivity and specificity of RUT increase to $100 \%, 4$ months after eradication.

Use of the antibody titer in the evaluation of $H$. pylori infection after eradication therapy involves a number of problems, such as a long period requires for the titer to become negative after successful eradication and relative judgment based on percent changes. However, the importance of this method will increase in the future to spread $H$. pylori eradication therapy widely because $\mathrm{E}$ plate method is convenient and can make reliable evaluation 8 weeks after eradication is achieved.

\section{References}

1) Marshall BJ, Goodwin CS, Warren JR, et al. Prospective double blind trial of duodenal ulcer relapse after eradication of Campylobacter pylori. Lancet 2: 1437-1442, 1988.

2) Malfertheiner P, Leodolter A, Peitz U. Cure of Helicobacter pylori associated ulcer disease through eradication. Ballieres Best Pract Res Clin Gastroenterol 14: 119-132, 2000.

3) Parsonnet J, Friedman GD, Vandersteen DP, et al. Helicobacter pylori infection and risk of gastric carcinoma. N Engl J Med 325: 1127-1131, 1991.

4) Montalban C, Manzaznal A, Boixeda D, Redondo C, Bellas C. Treatment of low-grade gastric MALT lymphoma with Helicobacter pylori eradication. Lancet 345: 798-799, 1995.

5) Furuta T, Kaneko E, Baba S, Arai S, Futami $H$. Percentage change in serum pepsinogens are useful as indices of eradication of Helicobacter pylori. Am J Gastroenterol 92: 84-88, 1997.

6) Fujioka T, Tokieda M. Validity of serum anti-Helicobacter pylori antibody using immnoassay for the diagnosis in eradication of Helicobacter pylori (in Japanese). Jpn J Med Pharm Sci 43: 573-579, 2000.

7) Cutler A, Schubert A, Schubert T. Role of Helicobacter pylori serology in evaluating treatment success. Dig Dis Sci 38: 2262-2266, 1993.

8) Kosunen TU, Seppala K, Sarna S, Sipponen P. Diagnostic value of decreasing IgG, IgA and IgM antibody titers after eradication of Helicobacter pylori. Lancet 339: 893-895, 1992.

9) Wang WM, Chen CY, Jan CM, et al. Long-term follow-up and serological study after triple therapy of Helicobacter pylori-associated duodenal ulcer. Am J Gastroenterol 89: 1793-1796, 1994.

10) Leung WK, Ng EK W, Chan FK L, Chung SC, Sung JJ. Evaluation of three commercial enzyme-linked immunosorbent assay kits for diagnosis of Helicobacter pylori in Chinese patients. Diagn Microbiol Infect Dis 34: 13-17, 1999.

11) Miwa $H$, Kikuchi $S$, Ohtaka $K$, et al. Insufficient diagnositic accuracy of imported serological kits for Helicobacter pylori infection in Japanese population. Diagn Microbiol Infect Dis 36: 95-99, 2000.

12) Vaira D, Malfertheiner P, Megraud F, et al, and European Helicobacter pylori HpSA Study Group. Noninvasive antigen-based assay for assessing Helicobacter pylori eradication: European multicenter study. Am J Gastroenterol 95: 925-929,2000.

13) Nishikawa K, Sugiyama T, Kato M, et al. A prospective evaluation of new rapid urease tests before and after eradication treatment of Helicobacter pylori, in comparison with histology, culture and 13C-urea breath test. Gastrointest Endosc 51: 164-168, 2000.

14) Murata H, Kawano S, Tsuji S, et al. Evaluation of the PyloriTek Test for detection of Helicobacter pylori infection in cases with and without eradication therapy. Am J Gastroenterol 93: 2102-2105, 1998. 